vache de 4 ans. Les filles de Carnation King Sylvia ne commenceront pas à rendre avant les derniers mois de l'année et par conséquent on ne peut juger à leur égard.

Ce qui précède donne une idée des procédés employés pour former le troupeau le plus productif que le monde ait jamais connu. L'élevage par race en a été le fondement; le reste a été accompli par une alimentation scientifique et par les soins les plus empressés.

Les jeunes filles de Avon Pontiac Echo et de Carnation King Sylvia seront croisées avec le fils de Matador Segis Walker;le sang de la race sera ainsi considérablement renforcé.

L'élevage méthodique des races a beaucoup fait dans le passé, mais il est bien possible qu'on ait dans l'avenir des vaches plus productives que celles de plein âge du troupeau actuel. Dorénavant, on emploiera les combinaisons qui ont donné les meilleurs résultats ; aujourd'hui, nous sommes heureux de constater que la production moyenne du troupeau s'accroît constamment, ce qui donne lieu à espérer qu'un rendement de $13.610 \mathrm{~kg}$. de lait par an ne sera plus considéré comme un fait phénoménal.

Elevage, nourriture et entretien sont nécessaires et - ne l'oubliez pas - le plus important, c'est l'élevage.

\title{
REVUE
}

\section{LA VALEUR ALIMENTAIRE DU LAIT PAR RAPPORT A SON PRIX DE VENTE}

PAR

J.-E. LUCAS,

Ingénieur agronome, Membre du Conseil supérieur de l'Agriculture. et

(1)

André LEROY,

Ingénieur agronome Chef de Travaux à l'Institut National Agronomique.

Le problème du ravitaillement en lait des grands centres urbains français se pose, depuis quelques années, d'une manière de plus en plus aiguë au commencement de chaque hiver. Cet état de choses est fort préjudiciable à l'hygiène générale, car non seulement le lait 
est l'aliment idéal de l'enfant, du malade et du vieillard, mais c'est encore un produit qui mériterait de tenir dans l'alimentation des adultes en bonne santé une place plus importante que celle qu'il occupe, en raison de sa haute valeur nutritive et des bons effets physiologiques de cerlains de ses constituants sur l'organisme humain. C'est done à bon escient que les Pouvoirs publics, et avec eux, nombre d'œuvres d'assistance à l'enfance se sont intéressés à celle grave question, afin d'en découvrir les causes, et d'y trouver les remèdes.

Malgré la hausse dont le lait a été l'objet pendant la guerre, hausse qui a atteint jusquà 0 fr. 70 par litre (écart entre 1 fr. 10, prix du litre de lait en décembre 1920 , et 0 fr. 40 prix normal de l'hiver 1913), c'est avec les plus grandes difficultés que les habitants de Paris et sa banlieue, notamment, peuvent se ravitailler en lait, surtout pendant la mauvaise saison. Alors qu'avant 1914, les apports quctidiens moyens, par voie terrée, du lait destiné à l'agglomérafion parisienne, atteignaient au total près de 900.000 litres, ces mêmes apports en 1919 ont, à grand peine, dépassé 560.000 litres, accusant ainsi une diminution de plus de $36 \%$.

Si l'on estime à 350.000 âmes environ l'accroissement de la population de la capitale au cours des hostilités, et si l'on évalue à 100.000 litres par jour la quantité de lait produite par les nourrisseurs parisiens et consommée sur place, en supplément du lait venu par chemin de fer, on peut en conclure que chaque habitant de Paris dispose, en moyenne, quotidiennement, de 01,147 , alors que la consommation correspondante avait été évaluée par l'un de nous, en 1910 , à 01,225 .

Les causes de cette crise sont multiples. Les principales d'entre elles sont, nous semble-t-il, le coût d'entretien élevé des vaches laitières, la difficulté de recrutement des vachers, les cours atteints sur les marchés par les fourrages, la vente facile et rémunératrice des beurres et des fromages, et enfin les poursuites judiciaires souvent injustifiées dont furent victimes un certain nombre de laitiers-nourrisseurs.

Actuellement, les frais d'entretien du bétail laitier soni extrèmement élevés. Si, pendant la belle saison, la production herbagère permet de nourrir à bon compte les animaux, il n'en est pas de même à partir du mois d'octobre, dès que les vaches retrouvent à l'étable leurs rations d'hiver à base de racines et de tourteaux. A 
titre documentaire, èt pour fixer les idées à ce sujet, nous estimons qu'une vache laitière coùte à un nourrisseur, installé à proximité de l'agglomération parisienne, une somme de $9 \mathrm{fr}$. environ par jour, compte tenu du prix des aliments, de la main-d'œuvre, de l'intérêt du capital engagé, ainsi que des aléas nombreux que présente l'entretien d'animaux coûteux, exposés à la contagion de maladies redoutables, comme la fièvre aphteuse et la tuberculose.

Si l'on admet qu'une bête produit en moyenne, par jour, 10 litres de lait (1), on voit que le prix de revient du litre, à la porte de la vacherie, est déjà de 0 fr. 90. D'autre part, le transport jusqu’à la crèmerie et la distribution à la clientèle occasionnent encore au producteur d'autres dépenses, qu'on peut chiffrer, au minimum, à 0 fr. 20 par litre, lorsque le lieu de vente se trouve, toutefois, à proximité de l'étable. Ces chiffres montrent que le laitier qui veut gagner $0 \mathrm{fr}$. 10 par litre, ce qui n'a rien d'exagéré, si l'on tient compte du travail fourni et de l'importance du capital engagé, est dans l'obligation de vendre son lait au moins 1 tr.20. Or, les grandes sociétés laitières vendaient, cet hiver, leur lait, à 1 fr. 10 le litre ; l'on est donc fondé à en déduire que les fournisseurs ordinaires de ces établissements ue retiraient pas de la production du lait un bénéfice suffisant, et l'on est tenté d'expliquer ainsi la défaveur dont cette production est actuellement l'objet de la part des agriculteurs.

La difficulté de recrutement du personnel spécialisé dans les soins à donner aux vaches laitières augmente encore, s'il est possible, cette délaveur. De tous les ouvriers de la ferme, le vacher est l'un des plus tenus.Les conditions pénibles de son existence ne sont pas en harmonie avec les goùts de lucre el de vie facile qui pénètrent les masses rurales, et l'on comprend ainsi la pénurie de vachers dont se plaignent, à juste titre, les fermiers, et qui conslitue l'un des plus grands obstacles à l'amélioration des conditions actuelles de la production du lait.

Sur les grands marchés urbains ou ruraux, les pailles, les fourrages, jusqu'à présent, se sont vendus fort cher. Aucune taxation efficace n'a limité la hausse des cours de ces denrées, dont les transactions obéissent et suivent encore la grande loi de l'offré et de la demande. Or, comme le lait, de son côté, était sévèrement taxé, il en est résulté que, dans bien des cas, par le jeu des lois économi-

(1) Une bète donnaut 3.600 litres de laịt peut être considérée comme une très bonne laitière. 
ques, il est devenu plus avantageux pour les fermiers d'écouler dans le commerce le foin récolté par eux plutôt que le faire consommer à leurs vaches. Rien ne saurait empêcher les agriculteurs qui, avec juste raison, cherchent le maximum de bénéfice, à se livres à ces opérations, s'ils y trouvent leur profit, et nous saisissons ainsi l'une des causes, et non des moindres, de la diminution de la production lactée.

Pas plus que les foins et les pailles, les beurres et les fromages n'ont été sévèrement taxés. L'agriculteur éloigné d'une grande ville a souvent trouvé plus d'avantages, pour cette raison, dans la transformation en produits fabriqués du lait qu'il expédiait en nature auparavant, vers les grandes villes. Lorsque ce beurre valait, pris à la ferme, $15 \mathrm{fr}$. le kilogramme, les sociétés de ramassage lui offrait d'acheter son lait à 0 fr. 70, c'est-à-dire sur une base inférieure à son prix de revient. Or, avec environ 25 litres de ce même lait, le même fermier pouvait obtenir 1 kilog. de beurre ce qui, au prix de $15 \mathrm{fr}$., fait ressortir le litre de lait à $0 \mathrm{fr}$. 60 , mais il conservait pour lui le lait écrémé et le babeurre, qu'il lui était permis de faire entrer dans les rations de ses porcs, et dont la valeur pour cet usage, étant donnés les cours élevés de la viande, pouvait être évaluée à 0 fr. 20 environ (1), il était donc plus avantageux, dans ces conditions, de transformer le lait en beurre, plutòt que de le vendre directement aux sociétés laitières ; le même raisonnement pourrait être repris, et discuté d'une manière analogue, en ce qui concerne la fabrication du fromage, mais nous estimons avoir suffisamment insisté sur cette quatrième cause de désaffection des fermiers pour les envois de lait en nature destinés à l'approvisionnement des grandes villes.

Enfin, à ces raisons économiques s'ajoute une cause d'ordre moral. La loi sur les spéculations illicites qui arme de pouvoirs véritablement draconiens, les juges d'instruction chargés de poursuivre ces affaires, a été appliquée avec unesévérité parfois déplacée. Dans certaines régions où les prix de vente du lait étaient à peine supérieurs à ceux d'avant-guerre, des laitierśs nourrisseurs ont été condamnés à des peines infamantes pour avoir tenté de mettre leurs

(1) D'après de nombreux résultats expérimentaux, les Américains admettent qu'au point de vue de l'engraissement des porcs, $100 \mathrm{kilog}$. de lait écrémé produisent le mème effet que $28 \mathrm{kilog}$. de grains (maïs, orge). Si l'on compte la valeur des céréales à $70 \mathrm{fr}$. le quintal, on voit que le litre de lait écrémé ressort sur cette base à $0 \mathrm{fr}$. 20 environ $(70 \times 28=19 \mathrm{fr}$. 16 pour 100 litres $)$. 
prix de vente un peu plus en harmonie avec leurs prix de revient, afin de faire cesser le déficit qui, en se prolongeant, n'aurait pas manqué de les conduire à la ruine. Or, ces hausses, poursuivies avec tant d'âpreté dans certaines juridictions, au point de prendre la forme tle persécutions véritables, étaient pärfaitement tolérées par les magistrals chargés de la surveillance d'une circonscription voisine. De tels écarts dans l'interprétation d'un même texte législatif n'ont pas manqué d'attirer sur leurs fàcheux effets l'attention des producteurs consciencieux, et ont ainsi contribué à détourner les agriculteurs d'un commerce devenu soudain si périlleux à exercer. Nous tenons à rappeler, toutefois, que les Pouvoirs publics, émus parles réclamations qui leur parvenaient, ont, depuis quelque temps, demandé aux juges de prendre l'avis, avaut de condamner les prétendus spéculateurs, des personnalités agricoles du département intéressé, et que cette intervention, fort heureusement, n'a pas tardé à produire, dans ce domaine, les plus salutaires effets.

En résumé, par ce qui précède, nous nous croyons londé à prétendre que la principale raison de la crise laitière est la suppression, par l'effet de l'intervention gouvernementale, du jeu normal de l'offre et de la demande, qui maintient pour le lait un prix de vente en harmonie, ni avec son prix de revient, ni avec sa valeur alimentaire.

Il nous a paru intéressant de donner comme preuve de ce qui précède, un tableau du prix de revient des 100 calories d'énergie nette, fournies à l'organisme humain par quelques-uns des aliments les plus courants. Nous avons dressè ce tableau en nous inspirant, d'une part, des chiffres empruntés aux travaux des physiologistes américains, Atwater et Bryant, et, d'autre part, des cours de détail pratiqués sur le marché d'un quartier de Paris très populeux, au printemps de l'année courante.

\begin{tabular}{|c|c|c|c|}
\hline Denrées & $\begin{array}{l}\text { Nombre de calories dans } \\
100 \text { gr. du produit } \\
\text { tel qu'il se trouve sur } \\
\text { le marché }\end{array}$ & $\begin{array}{c}\text { Prix } \\
\text { du kilog. }\end{array}$ & $\begin{array}{l}\text { Valeur vénale } \\
\text { des } \\
100 \text { calories }\end{array}$ \\
\hline
\end{tabular}

Viande de boeuf, morceaux à rôtir. .............

Viande de bouf, morceaux pour pot au feu........

Viande de veau, morceaux d̀ rotir...............

180

110

135
15 " 090

$7 \gg \quad 050$

$15 \% \quad 111$ 


\begin{tabular}{|c|c|c|c|}
\hline Denrées & $\begin{array}{c}\text { Nombre de calories dans } \\
100 \text { gr. du prortuit } \\
\text { tel qu'il se trouve sur } \\
\text { le marcbé }\end{array}$ & $\begin{array}{c}\text { Prix } \\
\text { du kilog. }\end{array}$ & $\begin{array}{c}\text { Valeur vénale } \\
\text { des } \\
100 \text { calories }\end{array}$ \\
\hline
\end{tabular}

Viande de mouton $\left\{\begin{array}{l}\text { épaule } \\ \text { gigot.. }\end{array}\right.$

Viande de porc, morceaux

à ròtir...............

Poulet.................

Hareng frais............

Eufs (par unité) ..........

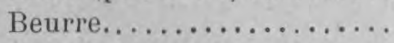

Lait..................

Fromage..............

Pain.................

Macaroni ..............

Sucre................

Chocolat...............

Huile blanche.... .......

Haricots secs ...........

Lentilles................

Pommes de terre........

Carottes ................

Pommes...............

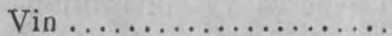

190 à 250

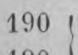

190

64

80

77

770

68

320

263

355

400

600

820

345

345

65

35

47

59
14 ×

070

090

234

032

$0 \quad 52$

$0 \quad 18$

$\begin{array}{lll}0 & 15\end{array}$

$0 \quad 31$

$\begin{array}{lll}0 & 0 \text { 's }\end{array}$

$\begin{array}{llll}0 & 11\end{array}$

008

$\begin{array}{lll}0 & 10\end{array}$

$\begin{array}{lll}0 & 07\end{array}$

$\begin{array}{lll}0 & 07\end{array}$

$0 \quad 11$

$0 \quad 05$

$0 \quad 11$

$0 \quad 25$

024

On voit, d'après les chiffres qui précèdent, que les aliments d'origine végétale sont moins coûteux que ceux d'origine animale, ce qui est parfaitement logique, car les constituants azotés des tissus végétaux n'ont pas la même valeur physiologique que ceux des produits animaux. Des calculs basés sur le pouvoir calorifique brut des matières dont l'homme se nourrit ne sauraient fournir, en effet, qu'une solution très grossière du problème de la détermination des valeurs alimentaires comparées de ces différents produits.

Les aliments servent à deux fins principales ; d'une part, ils sont destinés à fournir à l'organisme l'énergie dont il a besoin ( 3.300 calories par homme adulte soumis à un travail moyen, et par jour) ; d'autre part, ils servent à la réparation des pertes d'azote qu'entraine le fonctionnement de la machine animale. Pour réparer ces pertes, l'être humain doit trouver dans sa ration alimentaire une quantité suffisante de matières azotées.

Or, les aliments azotés sont ordinairement les plus coùteux, il y a donc pour l'homme un intérêt pratique à réduire au minimum 
compatible avec son maintien en bon état de santé la consommation de ces corps.

Les découvertes de la chimie physiologique ont montré que les constituants azotés des aliments courants, ne peuvent intégralement se substituer les uns aux autres dans les rations alimentaires de l'homme et des animaux supérieurs. Les recherches récentes, à la suite des travaux de Fischer et des savants de son école, ont contribué à rendre un peu moins mystérieuse la constitution intime de ces gros groupements d'atomes qui forment les corps azotés. Ces corps nous paraissent maintenant résulter de Y'union d'un certain nombre de fragments appelés acides-aminés, et caractérisés par le groupement atomique terminal $\mathrm{CHNH}^{2}-\mathrm{COOH}$. On a pu signaler jusqu'à présent dans les molécules albuminoïdes la présence de 18 acides aminés distincts.

L'action des sucs digestifs sur les protéiques opère lé dédoublement de ces corps en acides-aminés, comme la pioche du démolisseur dissocie les pierres d'un édifice. L'organisme animal s'empare de ces fragments libérés les uns des autres et les combine à nouveau entre eux de manière à opérer la synthèse de ses albumines spécifiques.

Les proportions des différents acides-aminés contenus dans les principales substances azotées alimentaires diffèrent essentiellement d'une de ces substances à l'autre, ainsi qu'il résulte de l'examen du tableau ci--après (page 18).

Ce tableau montre, tout d'abord, que la composition des matières albuminöïdes étudiées jusqu'à l'heure actuelle, n'est que partiellement connue. Une importante fraction de ces molécules, variant suivant les substances examinées entre 17 et $50 \%$, échappe encore à toute analyse.

Son observation suggère en outre, et c'est le point qui nous occupe particulièrement, que les albuminoïdes du lait sont notablement plus riches que toutes les autres en lysine et en tryptophane. Or, les expériences des savants américains Th. Osbonne, LafayetteMENDEL, tendent à prouver que ces deux acides-aminés sont particulièrement indispensable à l'accroissement pondéral du corps des vertébrés supérieurs ainsi qu'au maintien de l'équilibre de leur poids vif. L'arginine et l'histidine posséderaient également les mêmes propriétés, bien qu'à un moindre degré.

Enfin, l'examen des chiffres précédents, montre qu'il existe une 


\begin{tabular}{|c|c|c|c|c|c|c|c|}
\hline & $\begin{array}{l}\text { Caséine } \\
\text { du lait }\end{array}$ & Lactalbumine & $\begin{array}{l}\text { Albumine } \\
\text { de l'wui }\end{array}$ & $\begin{array}{l}\text { Viande } \\
\text { de bœnf }\end{array}$ & $\begin{array}{l}\text { Gluténine } \\
\text { du blé }\end{array}$ & $\begin{array}{l}\text { Gliadine } \\
\text { du blé }\end{array}$ & $\begin{array}{l}\text { Phaséoline } \\
\text { du haricot }\end{array}$ \\
\hline 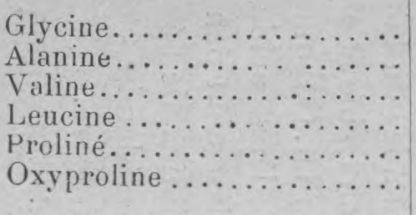 & $\begin{array}{r}0,0 \\
1,5 \\
7,2 \\
10,5 \\
6,7 \\
4,0\end{array}$ & $\begin{array}{r}0,0 \\
2,5 \\
0,9 \\
19,5 \\
4,0 \\
\end{array}$ & $\begin{array}{c}0,0 \\
2,22 \\
2,50 \\
10,71 \\
3,56 \\
》\end{array}$ & $\begin{array}{r}2.06 \\
3,72 \\
0,81 \\
11,65 \\
5.82 \\
\end{array}$ & $\begin{array}{l}0,89 \\
4,65 \\
6,24 \\
5,25 \\
4,23 \\
y\end{array}$ & $\begin{array}{c}0,0 \\
2,0 \\
3,34 \\
6,62 \\
13,22 \\
>\end{array}$ & $\begin{array}{l}0,55 \\
1,80 \\
104 \\
9,65 \\
2,77 \\
》\end{array}$ \\
\hline $\begin{array}{l}\text { Ac. aspartique............ } \\
\text { Ac. glutamique . . . . . . }\end{array}$ & 15,55 & $\begin{array}{r}1,0 \\
10,1\end{array}$ & $\begin{array}{r}2,20 \\
9,10\end{array}$ & $\begin{array}{r}4,51 \\
15,49\end{array}$ & $\begin{array}{r}0,91 \\
23,42\end{array}$ & $\begin{array}{r}0.58 \\
43,6 \hat{U}\end{array}$ & $\begin{array}{r}5,24 \\
14,54\end{array}$ \\
\hline $\begin{array}{l}\text { Phénylalanine........... } \\
\text { Tyrosine............ }\end{array}$ & 6,5 & $\begin{array}{l}2,6 \\
4,9\end{array}$ & $\begin{array}{l}5,07 \\
1,77\end{array}$ & $\begin{array}{l}3,15 \\
2,20\end{array}$ & $\begin{array}{l}1,97 \\
4,25\end{array}$ & $\begin{array}{l}2,35 \\
1,20\end{array}$ & $\begin{array}{l}3,25 \\
2,84\end{array}$ \\
\hline Sérine.............. & 0,5 & " & 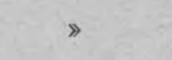 & $》$ & 0,74 & 0,13 & 0,38 \\
\hline $\begin{array}{l}\text { Histidine } \ldots \ldots \ldots \ldots \ldots \ldots \ldots \ldots \\
\text { Arginine. } \ldots \ldots \ldots \ldots \ldots \ldots \ldots \\
\text { Lysine } \ldots \ldots \ldots \ldots \ldots \ldots \ldots \ldots\end{array}$ & $\begin{array}{l}2,5 \\
4,8 \\
7,6\end{array}$ & $\begin{array}{l}1,5 \\
3,0 \\
8,1\end{array}$ & $\begin{array}{l}1,71 \\
4,91 \\
3,76\end{array}$ & $\begin{array}{l}1,76 \\
7,47 \\
7,59\end{array}$ & $\begin{array}{l}1,76 \\
4,72 \\
1,92\end{array}$ & $\begin{array}{l}0,61 \\
3,16 \\
0,75\end{array}$ & $\begin{array}{l}2,62 \\
4,87 \\
4,58\end{array}$ \\
\hline Tryptophane............ & 1,5 & 3,0 & présent & présent & présent & présent & présent \\
\hline Cystine..$\ldots \ldots \ldots \ldots \ldots$ & 0,1 & ” & $\gg$ & $\gg$ & 0,02 & 0,45 & $r$ \\
\hline 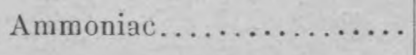 & 1,6 & 1,3 & 1,34 & 1,07 & 4,01 & 5,25 & 2,06 \\
\hline $\begin{array}{l}\text { Fraction connue de la molé- } \\
\text { cule } \ldots \ldots \ldots \ldots \ldots \ldots \ldots \ldots \ldots\end{array}$ & $71,38 \%$ & $62,10 \%$ & $48,85 \%$ & $67,20 \%$ & $59,68 \%$ & $82,69 \%$ & $56,19 \%$ \\
\hline
\end{tabular}


différence notable entre la structure intime des molécules albumi noïdes d'origine animale, et celle des protéines végétales. Parmi ces dernières, la phaséoline du haricot présente une teneur élevée en amino-acides essentiels (histinine, arginine, lysine) et possèderait pour cette raison une valeur alimentaire remarquablement élevée.

Donc, en raison de la composition intime de ses constituants azotés, le lait mérite une place à part parmi les aliments de l'homme. Cetle affirmation se renforce encore si l'on considère la haute teneur en vitamines de ce produit.

Les vitamines, ou facteurs accessoires de croissance, sont des composés de nature chimique inconnue qui existent en quantités variables dans la plupart de nos aliments, et dont la présence dans nos rations alimentaires est indispensable au bon état de notre organisme.

Un régime privé de vitamines est incompatible avec la vie. Tout animal soumis à une pareille alimentation perd l'appétit, s'amaigrit, et ne tarde pas à mourir après avoir présenté presque toujours des manifestations pathologiques cutanées ou nerveuses caractéristiques. Parmi ces vitamines, les unes favorisent la croissance, les autres ne permettent que le maintien en équilibre du poids du corps ; une troisième catégorie empêche la manifestation de maladies particulières appelées béri-béri, scorbut, ou pellagre, suivant les conditions dans lesquelles elles se manifestent et les accidents qu'elles produisent.

Les recherches auxquelles se sont livrés les savants américains, qui se sont occupés de la question, leur ont permis de classer nos aliments en deux grandes catégories suivant leưr richesse en vitamines.

La première catégorie est celle des aliments incomplets. Ces aliments ne renferment qu'une insuffisante quantité de ces facteurs accessoires. Parmi ceux-ci, il convient de citer les graines de céréales, les tubercules, les racines, les matières grasses animales constituant des dépôts de réserve sous-cutanés, les huiles végélales, et d'une façon générale, tout ce qui sert de matière de réserve, et n'est pas contenu dans des cellules vivant d'une vie aclive.

Les aliments complets, qui forment la seconde catégorie, sont riches en vitamines. A ce groupe appartiennent le lait, les fourrages 
verts, la graisse intra-cellulaire des viscères et, plus généralement, tous les tissus animaux ou végétaux qui vivent d'une vie active et tous les produits de sécrétion de cellules vivantes. On ne saurait trop insister sur celte curieuse constatation, qui oppose l'action biologique des produits jouant le rôle de réserves à celles des tissus vivants proprement dit, en période d'activité. Ces derniers constituent des aliments de protection, sans la présence desquels une ration ne saurait être physiologiquement équilibrée. Ainsi, par exemple, en aucun cas, une ration formée exclusivement de tubercules et de graines de céréales, tous organes de réserve, ne saurait suffire à entretenir la croissance et la vie d'un organisme animal.

Le lait est, au premier chef, un aliment de protection. Nul autre ne saurait lui disputer ce rang, et l'on s'explique ainsi pourquoi la crise de production dont cette denrée est actuellement l'objet, mérite de retenir, au premier chef, l'attention des hygiénistes.

C'est une erreur de croire que la consommation d'une importante quantité de viande est indispensable à l'homme moderne, même s'il exerce un métier manuel, dans les conditions actuelles de son existence.

Dans nombre de budgets modestes, des économies notables pourraient être réalisées, par le remplacement dans la ration alimentaire d'une certaine fraction de viande par une quantité isodyname de lait ou de produits de laiterie.

Prenons à titre d'exemple la ration moyenne journalière d'un habitant de Paris, d'après un travail statistique basé sur l'observation d'une période décennale et rapporté dans un travail récent de J. Alquien (1).

Cette ration comporte au total 2.700 calories (2) environ; $73 \%$ de cette quantité d'énergie sont fournis par des produits d'origine végétale, le reste provient d'aliments empruntés au règne animal; elle a la composition suivante:

(1) Conséquerices pratiques d'une alimentation scientifique. Bull. de la Société d'hygène alimentaire. Nos 4 et 5,1920 .

(2) Cette moyenne a été établie en tenant compte des aliments consommés d'une part, et, d'autre part, du nombre des habitants de la capitale, enfants compris. C'est pourquoi ce chiffre de 2.700 calories est inférieur aux 3.300 calories qui constituent la quantité d'énergie requise par un homme adulte. 


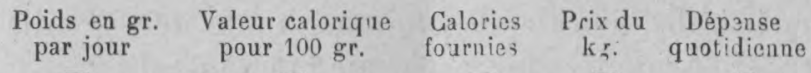

I. Aliments ori-

gine végétale:

Pain........ $\quad 420$

Légumes secs. $\quad 40$

Pommes de terre... 100

Légumes frais. 250

Sucre....... 40

Huile........ 10

Fruits frais.... 70

Vin ........ 332

II. Origine animale:

Viande ...... 200

Beurre, saindoux.... 18

Fromage..... 8

Lait.......... 213

Eufs......... 27

Poissons...... 40

263
345
65
30
400
820
50
60

$\begin{array}{r}1100 \\ 138 \\ 65 \\ 75 \\ 160 \\ 02 \\ 35 \\ 320 \\ \hline 195\end{array}$

$0 \lcm{42}$

$\begin{array}{lll}0 & 10\end{array}$

003

$0 \quad 50$

$\begin{array}{lll}0 & 12\end{array}$

006

$\begin{array}{lll}0 & 08\end{array}$

$\frac{061}{92}$

192
180

770

320

70

150

80

\begin{tabular}{|c|c|c|}
\hline 360 & $12 "$ & 240 \\
\hline 140 & $14 \Rightarrow$ & 025 \\
\hline 26 & $10 \gg$ & $\begin{array}{ll}0 & 08\end{array}$ \\
\hline 150 & $1 \gg$ & 021 \\
\hline 40 & 1/3 d'wuf & $\begin{array}{ll}0 & 15\end{array}$ \\
\hline 3 & $3 m$ & $\begin{array}{lll}0 & 12\end{array}$ \\
\hline & & $3 \longdiv { 2 1 }$ \\
\hline
\end{tabular}

L'on peut concevoir, sans qu'il en résulte de conséquences dommageables pour l'organisme, le remplacement de la viande figurant dans cette ration par une quantité isodyname de lait et de fromage. De cette manière la partie d'origine animale d'une semblable alimentation serait modifié comme il suit, sans que soit changée pour cela la quantité de matières azotées mises à la disposition de l'organisme :

\begin{tabular}{|c|c|c|c|c|}
\hline & Poids & Val. calorif. & Calories & $\begin{array}{c}\text { Dépenses } \\
\text { quotidiennes }\end{array}$ \\
\hline Beurre, saindoux.... & 18 & 770 & 140 & 025 \\
\hline Fromage....... & 25 & 320 & 80 & 025 \\
\hline Lait........... & 650 & 70 & 455 & 065 \\
\hline Eufs.......... & 27 & 150 & 40 & 015 \\
\hline \multirow[t]{2}{*}{ Poisson ....... } & 40 & 80 & 3 & $0 \quad 12$ \\
\hline & & & 718 & 142 \\
\hline
\end{tabular}


La généralisation de ce régime à toute une famille aurait donc une répercussion très favorable sur le budget de celle-ci.

Ajoutons que les nombreuses expériences réalisées dans cet ordre d'idées par les physiologistes ont prouvé que le régime lacto-végétarien constitue pour l'être humain l'un des plus hygiéniques qui soient.

Sans insister outre mesure sur cet aspect éconómique du problème de la consommation laitière, nous pensons que la recherche des remèdes appropriés à la solution de la crise actuelle possède une réelle importance sociale, puisque l'on peut conclure de ce qui précède que le lait devrait tenir une place plus importante que celle qui lui appartient dans l'alimentation des collectivités humaines.

Les mesures à adopter pour résoudre ce difficile problème peuvent appartenir à trois ordres différents. On peut envisager, en effet, dans ce but : $1^{\circ}$ des mesures de restriction; $2^{\circ}$ des mesures de taxation; $3^{\circ}$ le retour à la liberté commerciale.

Les restrictions à la consommation se sont bornées à l'interdiction de l'emploi du lait frais par les restaurants, pàtisseries et maisons de thé. Il s'agit plutôt, en la circonstance, d'une mesure prise pour frapper l'opinion publique, car les économies que son application stricte permet de réaliser sont infimes. Ce n'est donc pas dans cette voie qu’il faut travailler afin d'améliorer le ravitaillement en lait des grandes villes.

Faut-il alors recourir à la politique des taxations, comme l'ont envisagé certaines des personnalités qui se sont intéressées à la question (1), et limiter par exemple les fluctuations du prix du beurre et du fromage. Nous ne le croyons pas. Les essais fait antérieurement dans cet ordre d'idées ont échoué d'une manière si pitoyable qu'il paraît vain de songer à rétablir un régime ayant fourni de pareils résultats.

Une taxation sévère, bien loin de remplir son but qui est de régulariser les cours, amène une vente clandestine difficile à réprimer et conduit à une hausse des prix qui dépasse toutes proportions raisonnables, car les producteurs cherchent à se garantir par ces prix excessifs des risques qu'ils courent en vendant au-dessus de la taxe. D'autre part, si l'on empêche la vente des produits de laiterie, par des mesures inopportunes, l'on risque de provoquer l'abandon de la

(1) Voir Polack. - Approvis. en lait des grandes villes. Froid, déc, 1920, janv. 1921. Analysé dans le « Le Lait», p. 131, 1921. 
production laitière, car les éleveurs préféreront vendrc leurs fourrages ou les faire consommer à des animaux de boucherie, plutôt que de les faire entrer dans les rations des vaches laitières. Les Pouvoirs publics, pour rendre ses effets à la taxe sur les beurres et fromages, seraient donc amenés à appliquer de nouvelles taxes sur les foins, les pailles, la viande, c'est-à-dire à prendre toute une série de mesures dont l'application aurait pour but final de décourager complètement les agriculteurs et de diminuer leurs capacités de production.

Or, il ne faut pas l'oublier, ce n'est que par uneaugmentation de la production laitière à la ferme que l'on pourra assurer mieux le ravitaillement de nos grandes villes. Les bonnes mesures seront celles qui stimuleront le zèle des nourrisseurs, en leur permettant de réaliser le maximum de profits. C'est par le retour au jeu normal des lois économiques, par la suppression totale des taxations, que l'on obtiendra le retour à un état de chose normal, c'est-à-dire un approvisionnement suffisant du marché. Il faut donc rendre la liberté complète au commerce du lait, et consentir à payer pendant quelque temps cette denrée à des prix élevés.

En ce qui concerne le lait, l'application stricte d'une pareille politique ne manquerait pas d'avoir des inconvénients, car il faut songer aux sacrifices bien lourds qu'impose déjà aux pauvres gens le coût actuel du lait destiné à l'alimentation des enfants en bas âge. Mais c'est ici que devraient intervenir les Pouvoirs publics, et principalement les municipalités des grands centres urbains, en provoquant la création de "gouttes de lait » et autres œuvres d'assistance à l'enfance, et en subventionnant largement ces bienfaisantes institutions. Par cette voie charitable pourrait être atténuée la répercussion de la hausse, dont il faut d'ailleurs se garder d'exagérer l'importance, sur le budget des familles peu fortunées.

En résumé, le lait est peut-être de tous les aliments de l'espèce humaine, celui qui possède, au point de vue physiologique, le maximum de qualités. Ce n'est donc pas soutenir un paradoxe que de prétendre qu'il devrait être vendu plus cher, sil'on tient à ce que les agriculteurs s'irtéressent davantage à sa production. Ajoutons que, en échange de cette hausse, il deviendrait sans doute possible d'inciter les nourrisseurs à récolter leur lait dans des conditions de propreté suffisantes, et à éliminer de leurs étables les bêtes tuberculcuses. De la sorte, les agglomérations urbaines seraient assurées 
de recevoir chaque jour des quantités suffisantes de lait sain et propre et ces avantages réunis compenseraient amplement les légers sacrifices que devrait consentir en échange la communauté, au bénéfice des producteurs.

\section{BIBLIOGRAPHIE.}

Ouvrages consultés :

1. E. Lambling. - Précis de Biochimie, Masson, 1921.

2. R. Legendre. - Alimentation et ravitaillement, Masson Ed., 1920.

3. Mc Collum. - The Newer Knowledge in Nutrition, Me Millan Ed., 1920.

4. Sherman. - Food Products, Mc Millan Ed., 1920.

Publications périodiques :

1. J. Alquier. - Conséquences pratiques dune alimentation scientifique. Bull. Soc. d'Hyg. alim. Nos 4-5, 1920.

2. Edna Ferry. - Valeur alimentaire du lait, $38^{\mathrm{e}}$ rapport. Dairymens Association, 1919.

3. A. Leroy.- Théories nouvelles de l'alimentation. Bull. Soc, d'Hyg. alim., No 2,1920.

4. J.-E. Lucas. - Le ravitaillement de Paris en lait. Bull. Soc. nat. Agriculture, nov. 1911.

5. J.-E. Lucas. - Le ravitaillement de Paris avant et après là guerre, C. T. Acad. Agriculture, mars 1918.

6. J.-E. Lucas et André Leroy. - Sur le prix de revient du lait. Bull. de la Soc. des Agriculteurs de France, oct. 1920.

7. Th. Osbonne et Lafay, B. Mendel. - Jour. of Biolog. Chemistry, Baltimore, 1919-1920.

8. M. PolAcк. - Approvisionnement en lait des grandes agglomérations urbaines. Revue génér. Froid, déc. 1920, janv. 1921.

9. Schaeffer. - Les travaux sur les besoins qualitatifs d'azote et les vitamines. Ball. Soc. d'Hyg. alim., 1918. 